\title{
Synthesis of graphene on a Ni film by radio-frequency plasma-enhanced chemical vapor deposition
}

\author{
QI JunLei ${ }^{1}$, ZHANG LiXia $^{1}$, CAO Jian ${ }^{1}$, ZHENG WeiTao ${ }^{2 *}$, WANG Xin ${ }^{2}$ \& FENG JiCai ${ }^{1}$ \\ ${ }^{1}$ State Key Laboratory of Advanced Welding and Joining, Department of Materials Science and Engineering, Harbin Institute of Technology, \\ Harbin 150001, China; \\ ${ }^{2}$ Department of Materials Science, Key Laboratory of Mobile Materials, Ministry of Education, and State Key Laboratory of Superhard Materials, \\ Jilin University, Changchun 130012, China
}

Received December 5, 2011; accepted February 2, 2012; published online April 23, 2012

\begin{abstract}
Large-area single- or multilayer graphene of high quality is synthesized on Ni films by radio-frequency plasma-enhanced chemical vapor deposition (RF-PECVD) at a relatively low temperature $\left(650^{\circ} \mathrm{C}\right)$. In the deposition process, a trace amount of $\mathrm{CH}_{4}$ gas (2-8 sccm (sccm denotes standard cubic centimeter per minute at STP)) is introduced into the PECVD chamber and only a short deposition time (30-60 s) is used. Single- or multilayer graphene is obtained because carbon atoms from the discharging $\mathrm{CH}_{4}$ diffuse into the Ni film and then segregate out at its surface. The layer number of the obtained graphene increases when the deposition time or $\mathrm{CH}_{4}$ gas flow rate is increased. This investigation shows that PECVD is a simple, low-cost, and effective technique to synthesize large-area single- or multilayer graphene, which has potential for application as electronic devices.
\end{abstract}

graphene, PECVD, large-area

Citation: Qi J L, Zhang L X, Cao J, et al. Synthesis of graphene on a Ni film by radio-frequency plasma-enhanced chemical vapor deposition. Chin Sci Bull, 2012, 57: 3040-3044, doi: 10.1007/s11434-012-5120-4

Graphene is a two-dimensional crystalline sheet of carbon atoms packed into a honeycomb lattice that possesses a wealth of fascinating physical properties such as extremely high thermal conductivity, excellent transparency, tunable band gap, and high chemical and thermal stability [1-10]. In particular, graphene exhibits quantum electronic transport properties and extremely high mobility (about $15000 \mathrm{~cm}^{2} \mathrm{~V}^{-1} \mathrm{~s}^{-1}$ ) at room temperature, which is much higher than most conventional semiconductor materials [1-5]. Recently, much progress has been achieved in the fabrication of single- or multilayer graphene for use in nanoscale electronic applications including high-speed field-effect transistors, transparent electrodes, ultrasensitive sensors and electromechanical resonators [5-10].

For the purpose of electronic application, the synthesis of low-cost, large-area single- or multilayer graphene is highly desirable $[4,11]$. However, large-area graphene is difficult

*Corresponding author (email: wtzheng@jlu.edu.cn) to obtain using adhesive tape, mechanical cleavage and chemical exfoliation [12-14]. It has been reported that largearea graphene can be grown epitaxially on single crystal $\mathrm{SiC}$, but $\mathrm{SiC}$ substrates are expensive and it is difficult to exfoliate or transfer the graphene from $\mathrm{SiC}$ to another substrate because of the strong cohesive strength of the graphene/SiC interface and the extreme chemical stability of SiC [14-17]. Many researchers have reported the direct synthesis of large-area single- or multilayer graphene on transition metal substrates by chemical vapor deposition (CVD) [17-22]. An advantage of CVD is that graphene can be transferred to other substrates because some transition metals can be etched by acid solution [17,18]. However, the deposition temperatures required for thermal CVD are generally higher than $800^{\circ} \mathrm{C}$, and a relatively long deposition time is needed. The relatively high deposition temperature stems from the thermal decomposition of hydrocarbons. Therefore, it remains a challenge to develop a simple, effective and reproducible method to synthesize low-cost, high- 
quality and large-area graphene at relatively low temperature.

Recently, some researchers reported the synthesis of highquality large-area graphene using plasma-enhanced CVD (PECVD) at relatively low temperature. PECVD is probably the most promising method to produce graphene to date because it allows high-quality large-area graphene to be fabricated at low cost [14,23-25]. Also, graphene grown by PECVD exhibits a high carrier mobility, low sheet resistance and high optical transparency, suggesting it is of high quality and has great potential for various applications [14,23-25]. However, because the growth of graphene by PECVD is a recent development, further systematic research is required. In particular, the systematic study of the growth conditions required to produce different layers of graphene is limited. For example, the effects of deposition time and flow of reactive $\mathrm{CH}_{4}$ gas used during PECVD on the growth of graphene are not well understood.

In this work, we demonstrate that radio-frequency PECVD (RF-PECVD) can be used to synthesize high-quality, largearea single- or multilayer graphene on $\mathrm{Ni}$ films at a relatively low temperature of around $650^{\circ} \mathrm{C}$, and also explore the effect of deposition time and flow of reactive $\mathrm{CH}_{4}$ gas on the growth of graphene.

\section{Experimental}

A Ni film with a thickness of about $150 \mathrm{~nm}$ was deposited on a thermally-oxidized $\mathrm{Si}$ substrate using direct-current magnetron sputtering. The as-deposited Ni film was placed in the chamber of a PECVD system, and the pressure of the chamber was reduced to $5 \mathrm{~Pa}$. The $\mathrm{Ni}$ film was treated at $650^{\circ} \mathrm{C}$ for 40 min under $\mathrm{H}_{2}$ gas at a pressure of $200 \mathrm{~Pa}$. Graphene was synthesized on the Ni film using RF-PECVD at a frequency of $13.56 \mathrm{MHz}$ and substrate temperature of $650^{\circ} \mathrm{C}$ in a mixture of $\mathrm{CH}_{4}, \mathrm{Ar}$ and $\mathrm{H}_{2}$ gases (the gas flow was kept at 2-8, 80, and $40 \mathrm{sccm}$ for $\mathrm{CH}_{4}$, Ar, and $\mathrm{H}_{2}$, respectively). The total gas pressure was fixed at $1000 \mathrm{~Pa}$ by adjusting the gas outlet valve, and the power was $200 \mathrm{~W}$. The deposition time was 30-60 s. After deposition, the sample was rapidly cooled to room temperature at a cooling rate of about $10^{\circ} \mathrm{C} / \mathrm{s}$ by turning off the heater and filling the chamber with a flow of argon to promote carbon segregation and graphene formation $[15,17]$.

The obtained graphene samples were characterized by Raman spectroscopy (Renishaw Invia, Renshaw, UK) using an excitation wavelength of $514 \mathrm{~nm}$ and transmission electron microscopy (TEM, JEOL TEM-2010, JEOL, Japan) operating at $200 \mathrm{kV}$.

\section{Results and discussion}

Figure 1 shows the graphene sample synthesized by deposition

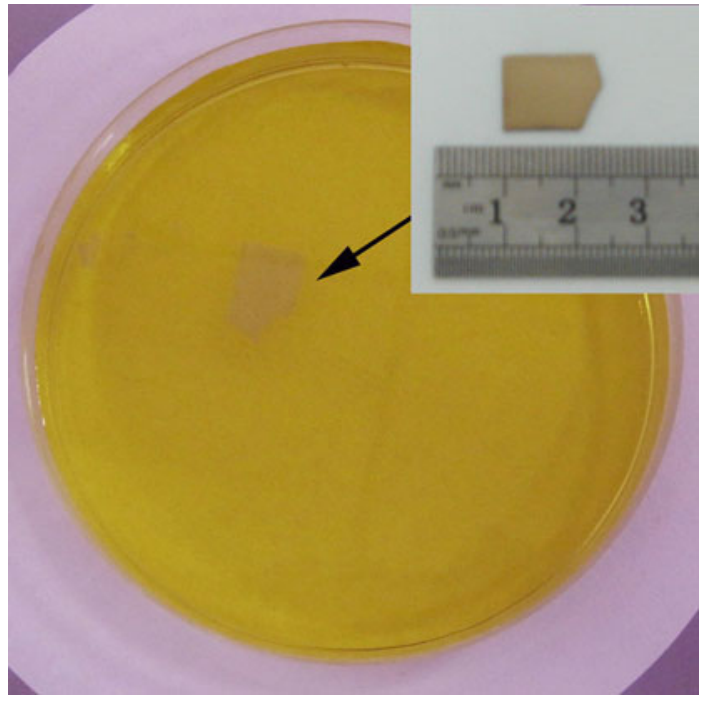

Figure 1 (Color online) A floating graphene sample obtained after etching the $\mathrm{Ni}$ layer in $1 \mathrm{~mol} / \mathrm{L} \mathrm{FeCl}_{3}$ aqueous solution, in which the inset shows the centimeter-scale graphene grown on the $\mathrm{Ni} / \mathrm{SiO}_{2} / \mathrm{Si}$ substrate. This graphene sample was synthesized with a deposition time of $30 \mathrm{~s}$.

for $30 \mathrm{~s}$ (the ratio of gas flow rates was kept at $\mathrm{CH}_{4} / \mathrm{Ar} / \mathrm{H}_{2}=$ 2/80/40 sccm). The graphene sample was obtained from floats on the surface of the $\mathrm{FeCl}_{3}$ solution following separation from the $\mathrm{Ni}$ film by wet etching in $\mathrm{FeCl}_{3}$ solution $(1 \mathrm{~mol} / \mathrm{L})[8,15]$. In the transfer process, the size and shape of the centimeter-scale graphene sample remains basically unchanged, as can be seen by comparing the image in Figure 1 to that in the inset of Figure 1. It is obvious that the size of the obtained graphene is dependent on the surface area of the $\mathrm{Ni}$ film, and that the graphene sample can easily be transferred to another substrate for the further evaluation and various applications $[4,15]$.

Raman spectra for graphene sample synthesized using various deposition times (the gas flow rate ratio was kept at $\mathrm{CH}_{4} / \mathrm{Ar} / \mathrm{H}_{2}=2 / 80 / 40 \mathrm{sccm}$ ) are presented in Figure 2. Raman spectroscopy is a powerful technique to characterize the crystalline quality and number of layers in a graphene sample [25]. The most notable feature for graphene is the appearance of the $2 \mathrm{D}$ peak, the position and shape of which are related to the formation and the layer number [15,16,25-28]. The graphene formed after deposition for $30 \mathrm{~s}$ exhibits a symmetric 2D peak at $\sim 2680 \mathrm{~cm}^{-1}$ with a full width at half-maximum of about $33 \mathrm{~cm}^{-1}$, which indicates that it is single-layer graphene [15,16,25-28]. As the deposition time is increased, the positions of the D and G peaks do not shift, but that of the $2 \mathrm{D}$ peak shifts obviously. The $2 \mathrm{D}$ peaks for the graphene samples grown in 40, 50 and $60 \mathrm{~s}$ appear around 2700, 2706 and $2713 \mathrm{~cm}^{-1}$, respectively. The reduced intensity of the $2 \mathrm{D}$ peak compared to $\mathrm{G}$ peak as the deposition time increases, suggests that the number of graphene layers increases with deposition time. Generally, the intensity ratio of the $\mathrm{D}$ peak to the $\mathrm{G}$ peak, denoted as the $R$-value, represents the amorphous phase content or the degree 


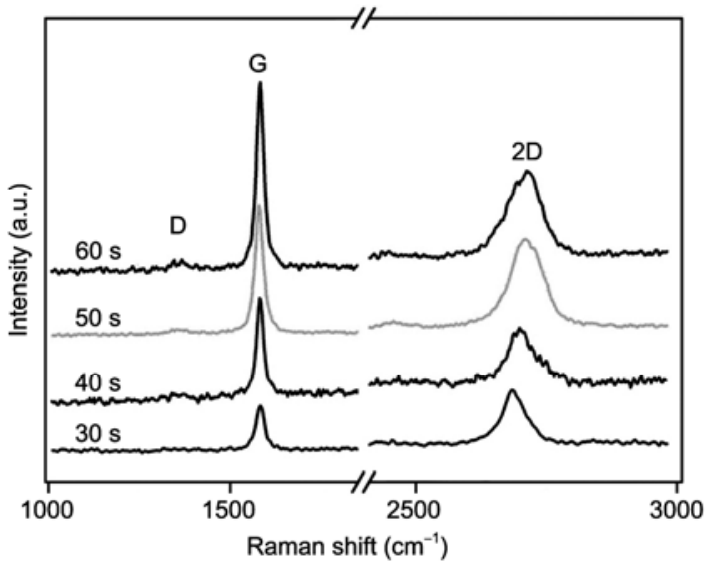

Figure 2 Raman spectra for graphene samples synthesized after various deposition times (the gas flow rate ratio was kept at $\mathrm{CH}_{4} / \mathrm{Ar} / \mathrm{H}_{2}=2 / 80 / 40$ sccm).

of crystallinity of carbonaceous materials. The smaller the $R$-value, the higher the degree of graphitization (or the fewer crystal defects present). The ratio of the high-intensity $G$ peak at $1580 \mathrm{~cm}^{-1}$ to the low-intensity $\mathrm{D}$ peak at $1350 \mathrm{~cm}^{-1}$ results in a very low $R$-value, which indicates that these graphene samples are of high quality with a high degree of graphitization and low number of crystal defects.

Figure 3(a) displays a TEM image of the graphene sample synthesized by deposition for $30 \mathrm{~s}$. The surface and edge of the obtained graphene are smooth. The selected area electron diffraction (SAED) pattern (inset of Figure 3(a)) corresponding to the square area in Figure 3(a) exhibits clear diffraction spots similar to those from mechanically cleaved graphene [26,29], indicating a graphite lattice structure with a good crystallinity. High resolution TEM (HRTEM) examination of the graphene sample confirms that the change in thickness corresponds to a few graphene layers. The HRTEM image in Figure 3(b) shows that a perfect single-layer graphene grew on the Ni film by deposition for $30 \mathrm{~s}$, which agrees well with Raman and SAED results. The HRTEM images in Figure 3(b) to (e) clearly show that the number of graphene layers increases with increasing deposition time. From the above results, it can be concluded that the deposition time significantly influences the number of graphene layers.

Raman spectra for the graphene samples synthesized using different ratios of gas flow rate at a constant deposition time of $30 \mathrm{~s}$ and temperature of $650^{\circ} \mathrm{C}$ are shown in Figure 4. The Raman spectra for the graphene samples deposited using $\mathrm{CH}_{4}$ flow rates of 2, 4, 6 and $8 \mathrm{sccm}$ show that the graphene sample contain approximately one layer $(1 \mathrm{~L})$, three layers $(3 \mathrm{~L})$, five layers $(5 \mathrm{~L})$ and ten layers $(10 \mathrm{~L})$, respectively, indicating that the number of graphene layers increases with increasing $\mathrm{CH}_{4}$ gas flow rate. In the syntheses of carbon materials such as nanotubes and nanosheets by PECVD, the ratio of gas flow rate significantly affects the morphology and microstructure of the carbon nano-

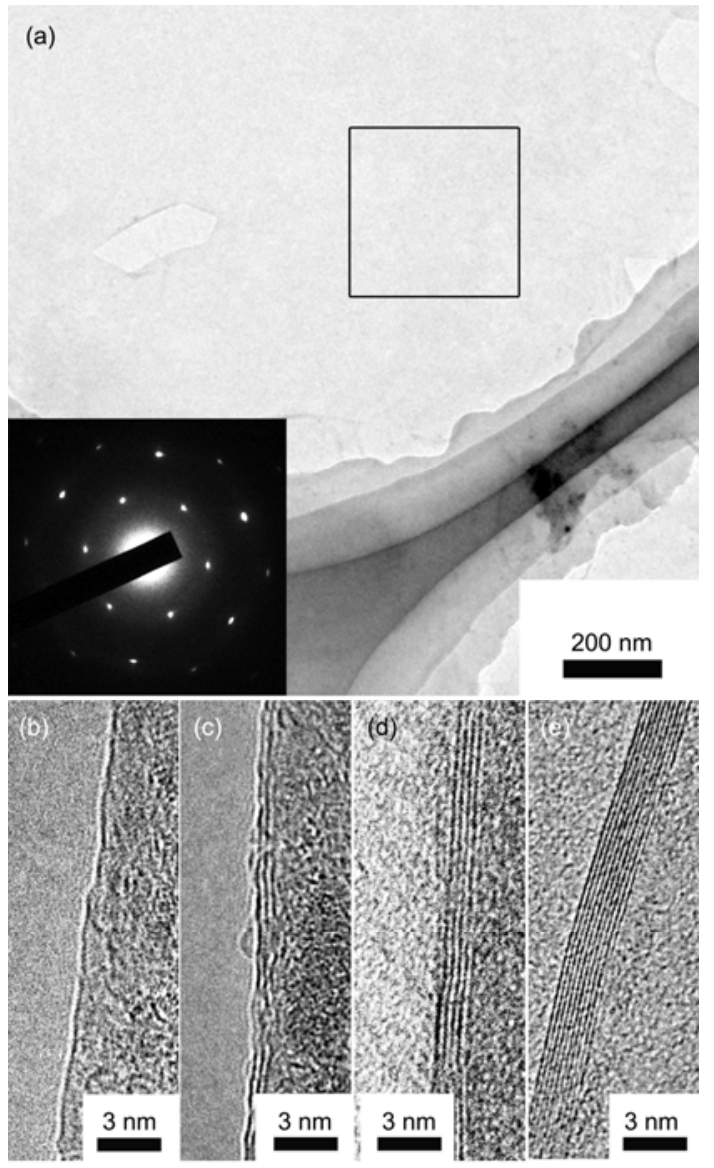

Figure 3 (a) A typical TEM image for the graphene sample grown in $30 \mathrm{~s}$, in which the inset shows the SAED pattern for the sample. HRTEM images of graphene samples synthesized using various deposition times: (b) 30, (c) 40, (d) 50 and (e) $60 \mathrm{~s}$

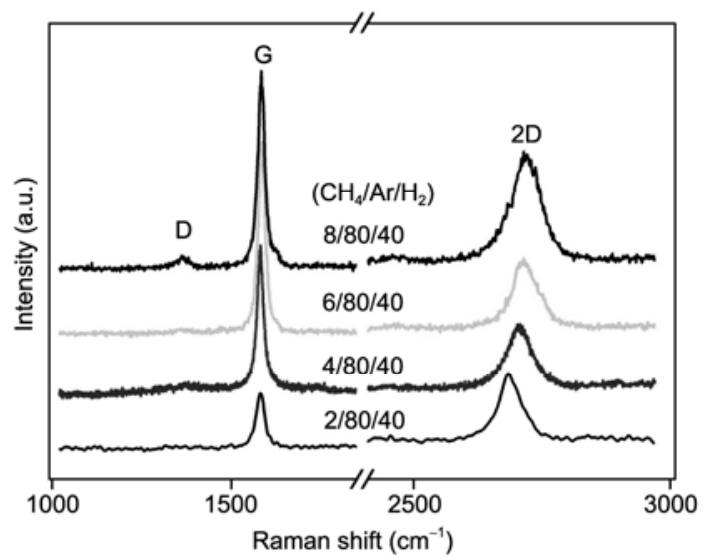

Figure 4 Raman spectra for graphene samples synthesized using various gas flow rate ratios (deposition time: $30 \mathrm{~s}$ and temperature: $650^{\circ} \mathrm{C}$ ).

material, and especially, the concentration of carbon source gas is important [25,30,31]. In Figure 4, the two Raman peaks of the highest intensity are the $\mathrm{G}$ band at $\sim 1580 \mathrm{~cm}^{-1}$ and 2D band at 2680 to $2718 \mathrm{~cm}^{-1}$, while the $\mathrm{D}$ band at $\sim 1350 \mathrm{~cm}^{-1}$ is very weak. The 2D peak in the Raman spectrum 


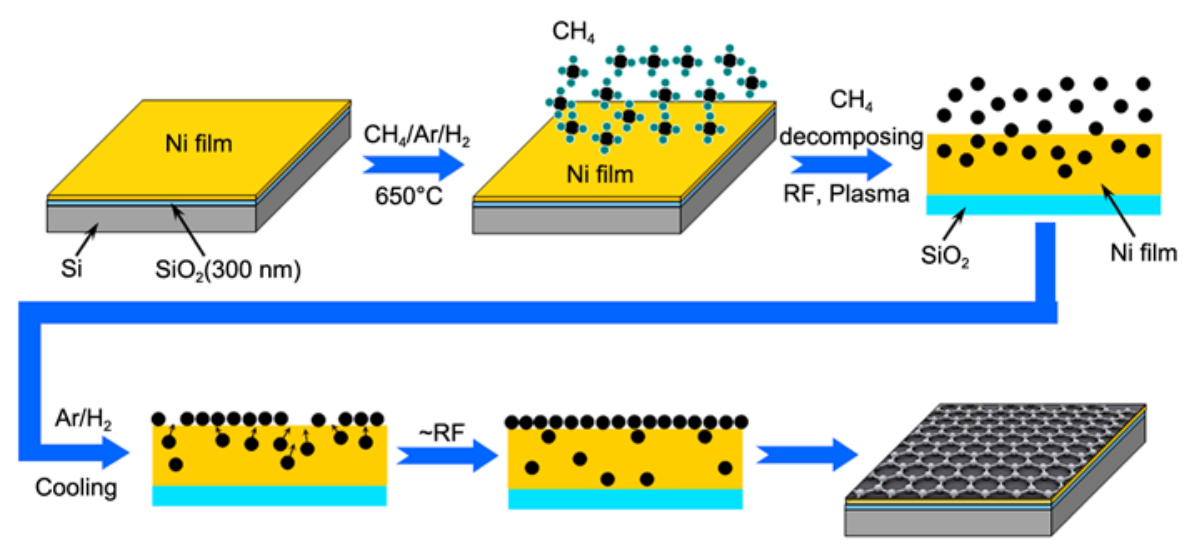

Figure 5 (Color online) Schematic illustration of the formation process of graphene.

for the samples deposited using a $\mathrm{CH}_{4}$ gas flow rate of 2, 4, 6 and $8 \mathrm{sccm}$, appears around 2680, 2705, 2712 and 2719 $\mathrm{cm}^{-1}$, respectively. According to previous reports and our above description, the obtained graphene layer deposited using a $\mathrm{CH}_{4}$ gas flow rate of 2, 4, 6 and $8 \mathrm{sccm}$ is about 1, 5, 10 and $>10 \mathrm{~L}$. The Raman spectra indicate that the number of graphene layers increase with increasing the $\mathrm{CH}_{4}$ gas flow rate, suggesting the graphene deposited using a lower $\mathrm{CH}_{4}$ gas flow rate is of higher quality and possesses a lower degree of crystal defects. These results mean that the thickness of graphene prepared by RF-PECVD can be controlled by the ratio of $\mathrm{CH}_{4}$ flow rate in a mixture of $\mathrm{CH}_{4} / \mathrm{Ar} / \mathrm{H}_{2}$ gases.

Based on the above results, it follows that PECVD is a very powerful technique for the synthesis of large-scale graphene at relatively low temperature in a short time. In PECVD, plasma generated by electrical discharges is more effective at decomposing reactive gas than that in conventional CVD. With the assistance of plasma, reactive gas species (radicals) can be generated in high density at relatively low temperature [32-35]. Correspondingly, graphene can be prepared at low temperature in a short time. The process of graphene growth on the Ni film can be explained as follows: an extremely high concentration of reactive radicals is formed at lower temperature and pressure because of the assistance of plasma [34,35]. This means that the trace amount of $\mathrm{CH}_{4}$ introduced into the PECVD chamber within a short time will produce a high concentration of carbon reactive radicals. During PECVD of graphene, the carbon atoms from decomposing $\mathrm{CH}_{4}$ gas enter the interior of the $\mathrm{Ni}$ film. Upon cooling, the carbon atoms leave the interior of the Ni film, and segregate on its surface to form graphene because the solubility of carbon in $\mathrm{Ni}$ is temperature-dependent $[15,17,18]$, as is schematically illustrated in Figure 5. In addition, an increase in the fraction of $\mathrm{CH}_{4}$ in the mixture of gases also leads to an increased concentration of reactive carbon radicals [36]. Thus, the thickness of graphene can be controlled by either deposition time or $\mathrm{CH}_{4}$ flow rate in the mixture of reaction gases.

\section{Conclusions}

We have demonstrated a low-temperature synthesis of highquality, large-area single- or multilayer graphene on $\mathrm{SiO}_{2} / \mathrm{Si}$ substrates covered with a Ni film using RF-PECVD. The number of graphene layers increases by increasing either deposition time or the ratio of $\mathrm{CH}_{4}$ gas flow rate in the mixture of reaction gases. Single-layer graphene can be synthesized at a substrate temperature as low as $650^{\circ} \mathrm{C}$ with a gas flow rate ratio of $2 / 80 / 40 \mathrm{sccm}\left(\mathrm{CH}_{4} / \mathrm{Ar} / \mathrm{H}_{2}\right)$, and it can be easily transferred to other substrates by wet-etching Ni. These results show that RF-PECVD is a simple, lowcost, highly-effective and reproducible technique to synthesize graphene at relatively low temperature compared to conventional thermal CVD.

This work was supported by the National Natural Science Foundation of China (51105108), the Fundamental Research Funds for the Central Universities (HIT. NSRIF. 2010113 and 2010115), and the Foundation of the Key Laboratory for Advanced Materials Processing Technology, Ministry of Education (2010007).

1 Novoselov K S, Geim A K, Morozov S V, et al. Electric field effect in atomically thin carbon films. Science, 2004, 306: 666-669

2 Geim A K, Novoselov K S. The rise of graphene. Nat Mater, 2007, 6: 183-191

3 Chen Z P, Ren W C, Gao L B, et al. Three-dimensional flexible and conductive interconnected graphene networks grown by chemical vapour deposition. Nat Mater, 2011, 10: 424-428

4 Li X, Cai W, An J, et al. Large-area synthesis of high-quality and uniform graphene films on copper foils. Science, 2009, 324: 1312-1314

5 Tang Y B, Lee C S, Chen Z H, et al. High-quality graphenes via a facile quenching method for field-effect transistors. Nano Lett, 2009, 9: $1374-1377$

6 Levchenko I, Volotskova O, Shashurin A, et al. The large-scale production of graphene flakes using magnetically-enhanced arc discharge between carbon electrodes. Carbon, 2010, 48: 4570-4574

7 Gilje S, Song H, Wang M, et al. A chemical route to graphene for device applications. Nano Lett, 2007, 7: 3394-3398

8 Bunch J S, van der Zande A M, Verbridge S S, et al. Electromechanical resonators from graphene sheets. Science, 2007, 315: 490-493 
9 Schedin F, Geim A K, Morozov S V, et al. Detection of individual gas molecules adsorbed on graphene. Nat Mater, 2007, 6: 652-655

10 Eda G, Lin Y Y, Miller S, et al. Transparent and conducting electrodes for organic electronics from reduced graphene oxide. Appl Phys Lett, 2008, 92: 233305

11 Liu F, Zhang Y. Substrate-free synthesis of large area, continuous multi-layer graphene film. Carbon, 2010, 48: 2394-2400

12 Wu Z S, Ren W C, Gao L B, et al. Synthesis of high-quality graphene with a pre-determined number of layers. Carbon, 2009, 47: 493-499

13 Juang Z Y, Wu C Y, Lo C W, et al. Synthesis of graphene on silicon carbide substrates at low temperature. Carbon, 2009, 47: 2026-2031

14 Qi J L, Zheng W T, Zheng X H, et al. Relatively low temperature synthesis of graphene by radio frequency plasma enhanced chemical vapor deposition. Appl Surf Sci, 2011, 257: 6531-6534

15 Kim K S, Zhao Y, Jang H, et al. Large-scale pattern growth of graphene films for stretchable transparent electrodes. Nature, 2009, 457: 706-710

16 Berger C, Song Z M, Li X B, et al. Electronic confinement and coherence in patterned epitaxial graphene. Science, 2006, 312: 11911196

17 Yu Q, Lian J, Siriponglert S, et al. Graphene segregated on Ni surfaces and transferred to insulators. Appl Phys Lett, 2008, 93: 113103

18 Reina A, Jia X, Ho J, et al. Large area, few-layer graphene films on arbitrary substrates by chemical vapor deposition. Nano Lett, 2009, 9: 30-35

19 Coraux J, N'Diaye A T, Busse C, et al. Structural coherency of graphene on $\operatorname{Ir}(111)$. Nano Lett, 2008, 8: 565-570

20 Sutter P W, Flege J I, Sutter E A. Epitaxial graphene on ruthenium. Nat Mater, 2008, 7: 406-411

21 Vázquez de Parga A L, Calleja F, Borca Jr B, et al. Periodically rippled graphene: Growth and spatially resolved electronic structure. Phys Rev Lett, 2008, 100: 056807

22 Pan Y, Zhang H G, Shi D X, et al. Highly ordered, millimeter-scale, continuous, single-crystalline graphene monolayer formed on $\mathrm{Ru}(0001)$. Adv Mater, 2009, 21: 2777-2780

23 Volotskova O, Levchenko I, Shashurin A, et al. Single-step synthesis and magnetic separation of graphene and carbon nanotubes in arc discharge plasmas. Nanoscale, 2010, 2: 2281-2285

24 Nandamuri G, Roumimov S, Solanki R. Remote plasma assisted growth of graphene films. Appl Phys Lett, 2010, 96: 154101

25 Kim Y, Song W, Lee S Y, et al. Low-temperature synthesis of graphene on nickel foil by microwave plasma chemical vapor deposition. Appl Phys Lett, 2011, 98: 263106

26 Ferrari A C, Meyer J C, Scardaci V, et al. Raman spectrum of graphene and graphene layers. Phys Rev Lett, 2006, 97: 187401

27 Malard L M, Pimenta M A, Dresselhaus G, et al. Raman spectroscopy in graphene. Phys Rep-Rev Sect Phys Lett, 2009, 473: 51-87

28 Pollard A J, Nair R R, Sabki S N, et al. Formation of monolayer graphene by annealing sacrificial nickel thin films. J Phys Chem C, 2009, 113: 16565-16567

29 Meyer J C, Geim A K, Katsnelson M I, et al. The structure of suspended graphene sheets. Nature, 2007, 446: 60-63

30 Chhowalla M, Teo K B K, Ducati C, et al. Growth process conditions of vertically aligned carbon nanotubes using plasma enhanced chemical vapor deposition. J Appl Phys, 2001, 90: 5308-5317

31 Wang J J, Zhu M Y, Outlaw Ron A, et al. Synthesis of carbon nanosheets by inductively coupled radio-frequency plasma enhanced chemical vapor deposition. Carbon, 2004, 42: 2867-2872

32 Terranova M L, Sessa V, Rossi M. The world of carbon nanotubes: An overview of CVD growth methodologies. Chem Vapor Depos, 2006, 12: 315-325

33 Kim J, Ishihara M, Koga Y, et al. Low-temperature synthesis of largearea graphene-based transparent conductive films using surface wave plasma chemical vapor deposition. Appl Phys Lett, 2011, 98: 091502

34 Okita A, Suda Y, Ozeki A, et al. Predicting the amount of carbon in carbon nanotubes grown by $\mathrm{CH}_{4} \mathrm{rf}$ plasmas. J Appl Phys, 2006, 99: 014302

35 Denysenko I, Ostrikov K. Ion-assisted precursor dissociation and surface diffusion: Enabling rapid, low-temperature growth of carbon nanofibers. Appl Phys Lett, 2007, 90: 251501

36 Meyyappan M, Delzeit L, Cassell1A, et al. Carbon nanotube growth by PECVD: A review. Plasma Sources Sci Technol, 2003, 12: 205-216

Open Access This article is distributed under the terms of the Creative Commons Attribution License which permits any use, distribution, and reproduction in any medium, provided the original author(s) and source are credited. 